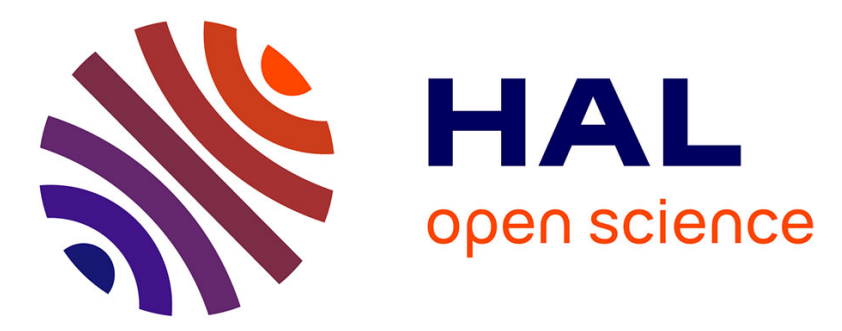

\title{
Current status and challenges of the modeling of organic photodiodes and solar cells
}

\author{
Raphael Clerc, B Bouthinon, A M Mohankumar, Patrice Rannou, J Vaillant, \\ T Maindron, B Racine, Y-F Chen, L Hirsch, J M Verilhac, et al.
}

\section{- To cite this version:}

Raphael Clerc, B Bouthinon, A M Mohankumar, Patrice Rannou, J Vaillant, et al.. Current status and challenges of the modeling of organic photodiodes and solar cells. International Electron Device Meeting 2016, Dec 2016, San Francisco, United States. hal-01385518

\author{
HAL Id: hal-01385518 \\ https://hal.science/hal-01385518
}

Submitted on 21 Oct 2016

HAL is a multi-disciplinary open access archive for the deposit and dissemination of scientific research documents, whether they are published or not. The documents may come from teaching and research institutions in France or abroad, or from public or private research centers.
L'archive ouverte pluridisciplinaire HAL, est destinée au dépôt et à la diffusion de documents scientifiques de niveau recherche, publiés ou non, émanant des établissements d'enseignement et de recherche français ou étrangers, des laboratoires publics ou privés. 


\title{
Current status and challenges of the modeling of organic photodiodes and solar cells
}

\author{
Invited paper \\ R. Clerc ${ }^{1}$, B. Bouthinon ${ }^{2}$, M. Mohankumar ${ }^{3}$, P. Rannou ${ }^{3}$, J. Vaillant ${ }^{4}$, T. Maindron ${ }^{4}$, B. Racine ${ }^{4}$, \\ Y-F Chen ${ }^{5}$, L. Hirsch ${ }^{5}$, J. M. Verilhac ${ }^{6}$, A. Pereira ${ }^{6}$, A. Revaux ${ }^{6}$.
}

1 Univ. de Lyon, UJM-Saint-Etienne, Institut d'Optique Graduate School, CNRS, UMR5516, Lab. Hubert Curien, Saint-Etienne France, email: raphael.clerc@ institutoptique.fr, 2 ISORG, Grenoble, France, 3 UMR5819-SPrAM (CEA/CNRS/UGA), INAC, CEA-

Grenoble, France 4 CEA LETI Grenoble France, 5 Univ. Bordeaux, IMS, UMR CNRS 5218, Talence, France 6 CEA, LITEN, Grenoble, France

\begin{abstract}
Progress in the modeling of charge transport in solution processed solar cells and photodiodes is reviewed. Through several examples involving modeling and original experiments, the role of intentional doping, structural defects, and oxygen contamination are discussed.
\end{abstract}

\section{INTRODUCTION}

Organic electronics is an emerging technology using $\pi$ conjugated molecules or polymers (i.e. organic semiconductors) [1] to produce electronic devices. Batteries, photovoltaic, (bio) sensors, displays, lighting are among the most relevant fields of applications of this technology [2-4]. One particular challenge of this domain consists in taking advantage of solution-processed organic materials to produce low-cost and large area devices by either roll-to-roll, sheet-tosheet or printing technologies [3]. In this context, solution processed photodiodes are of particular interest, either for outdoor [5-6] and indoor [7] photovoltaic or imaging applications [8-10]. In the field of solar energy, External Power Conversion Energy (EPCE) exceeding $10 \%$ have been reported using Bulk HeteroJunction (BHJ) polymer based devices [6] (approaching its fundamental limits [11]) and more than $20 \%$ using solution processed hybrid inorganic/organic perovskite semiconductors [12]. Organic imagers are also attractive, either to improve silicon CMOS imagers [13], or to realize large-area, flexible, low-weight full organic imagers. Dark current level comparable with silicon photodiodes have already been reported [14][15]. In particular, large-area organic imagers, either deposited on the top of a thin-film transistor (TFT) or organic transistor backplane are attractive alternatives to amorphous silicon imagers in X-ray detectors for medical applications [9][16]. In all cases, despite the instability of most organic material in presence of UV, water and/or oxygen, the reliability of polymer-based devices has greatly improved and is no longer considered as a major obstacle, provided that suitable materials, processes, and encapsulations are used [17][18].

With the emergence of this technology, device modeling is more and more needed, either to support device processing or to predict system performances. The aim of this paper is to review the existing physical models, to discuss the main ingredients needed for device simulations, and to give example of application of modeling, in close comparison with experiments.

\section{CHARGE TRANSPORT IN DISORDERED ORGANIC SEMICONDUCTORS.}

It is generally admitted that $\pi$-conjugated small molecules molecules and polymers behave as disordered semiconductors [19], even if the exact origins of such disorder are still under debate, and are material/process dependent. Structural heterogeneities in amorphous or polycrystalline semiconductors, induced by processes and interfaces, are of course a major sources of disorder, and many studies have reported significant mobility improvements by optimization of the material morphology [20]. However, it seems that disorder may have also intrinsic origins, induced either by chemical impurities, by changes in the surrounding polarization during charge transport [19], or as recently proposed, induced by large thermal molecular motions (transient localization), a direct consequence of the weak strength of van der Waals intermolecular interactions [21]. This later theory is supposed to explain the low temperature mobility improvement reported in some high mobility $\left(>1 \mathrm{~cm}^{2} \cdot \mathrm{V}^{-1} \cdot \mathrm{s}^{-1}\right)$ organic single crystals, and sometimes referred as "band like" transport [22].

In the literature, two main approaches have been used to model transport in disordered semiconductor. The first one consists in solving the hopping master equation, either by Monte Carlo [23] or by direct solving [24]. Quantum chemistry can be used to calculate hopping site energy distributions [25]. In many cases however, the disordered energy positions of hopping sites is assumed to follow a Gaussian law, and hopping probabilities are modeled by Miller-Abrahams expressions, leading to the so-called Gaussian Disorder Model (GDM) [19]. This model predicts an increasing mobility with temperature, electric field and carrier concentrations, in agreement with several experiments [26]. There are exceptions, such as P3HT [27] or the high mobility "band like" single crystal for instance [22]. Numerically expensive, this approach is typically used to get insight on transport [26], recombinations [29] noise properties [24] or blend morphology [28] of organic materials (rather than to simulate complete device).

An illustration of the capability of GDM models to reproduce a large set of experimental data is shown in Fig. 15 . In these experiments, several asymmetrical $\alpha$-NPB p-only devices have been realized by evaporation, featuring different thicknesses in the $20-110 \mathrm{~nm}$ range. $\alpha$-NPB is a hole transport material (HTM), classically used in OLED [30]. Interestingly, 
the conductivity of $\alpha-\mathrm{NPB}$ has been tuned by molecular doping of $\mathrm{MoO}_{3}$, as in [31]. In agreement with GDM theory, the conductivity shows a clear exponential dependency with doping concentration (Fig.2), despite significant process induced variability. Moreover, using low-field conductivity extracted on each device, I-V experiments can be satisfactory reproduced with the same set of GDM parameters, provided that the mobility electric-field dependence is included [30].

\section{DRIFT DIFFUSION (DD) MODELS FOR ORGANIC SEMICONDUCTOR DEVICES.}

The second commonly used approach to model organic device is the well-known Drift Diffusion model, numerically efficient and versatile. Disorder is included via Gaussian Bands (or exponential bandtails), shallow and deep traps and GDM inspired mobility models, as it was done in the past for amorphous silicon solar cells [32]. It can account for electron and holes transport in heterostructures [33] and interface charges and dipoles [30], which both play an important role in organic devices. Light propagation is performed by solving Maxwell equations [34]. DD requires detailed calibration with experiments, and especially band structure parameters (using UPS), mobility (using Time of Flight TOF), optical index and recombination (deep and shallow traps, bandtails). Even if the applicability of DD to model organic semiconductors is questionable [35], the efficiency of this approach to tackle many sophisticated phenomena such as transient low-light intensity in organic photodiodes [36], impact of blend morphology in BHJ solar cell [37][38], role of electron/hole transport layer (sometimes causing $S$ shape [39]), analysis of impedance spectroscopy experiments [40] (and many others) is remarkable.

In that respect, let us give a recent example illustrating the strengths and weaknesses of the drift diffusion model (Fig. 68 ). The p-only polycrystalline perovskite $\mathrm{MAPbI}_{3}$ has been processed and characterized at low temperature. Accounting for volume and interface shallow traps, I-V curves at $10 \mathrm{~K}$ have been nicely fitted by a simple DD model (Fig.7), extracting data from electroluminescence, $\mathrm{I}-\mathrm{V}$, TOF and $\mathrm{C}-\mathrm{V}$ experiments (not shown here). Interestingly, with no additional fitting parameters, the model also correctly predicts the higher temperature I-V curves, however with a lower accuracy (Fig.8). A closer look to experiments has indeed revealed that the experimental temperature activated conductivity was in better agreement with hopping models, suggesting the occurrence of transport from traps to traps, not included in the DD model.

\section{AN EXAMPLE OF DEVICE SIMULATION: FRONT AND BACK SIDE QUANTUM EFFICIENCY DIFFERENCES IN SEMI-TRANSPARENT ORGANIC PHOTODIODES CAUSED BY OXYGEN CONTAMINATION}

To conclude, let us give another example of the capability of device simulation to support device processing. Semitransparent organic photodiodes may feature significantly different external quantum efficiency (EQE) when measured from front and back sides (see Fig. 9-13). It was initially suspected that this difference was caused by the different optical properties of top and bottom layers. However simulations has shown that optics can hardly explain of fraction of this discrepancy, and not its thickness and voltage dependency. On the other hand, the introduction of deep acceptor traps, inducing a negative charge within the active layer in presence of light generated carriers was able to explain this effect (Fig. 11\&12). In fact, the introduction of a single level acceptor deep traps, inducing both recombination and space charge effects, was able to reproduce a large set of experimental data (Fig.12). As oxygen is known to potentially induce negative charges and trapping [41], new experiments have been performed in absence of oxygen. In this later case, in agreement with simulations (Fig. 13), the difference of EQE has almost disappeared. The comparison between experiments and DD simulations has thus been of great help, indicating oxygen contamination as the potential origin of this effect.

\section{Conclusions}

Despite the complexity of disordered organic materials, Drift Diffusion based device simulation remains a powerful tool to analyze organic solar cells and photodiodes, and in particular the role of intentional doping, structural or induced defects states and interfaces. It requires however experimented users, capable on one hand to improve models thanks to the progress of more sophisticated approaches (such as variable range hopping model and quantum chemistry) and on the other hand, to perform detailed calibrations with experiments.

\section{ACKNOWLEDGMENT}

This work benefited from the support of the project TAPIR ANR15CE24002405 of the French National Research Agency.

\section{REFERENCES}

[1] A. Facchetti, "П-conjugated polymers for organic electronics and photovoltaic cell applications". Chem. Mater., 23(3), 733-758. (2010).

[2] D. Lupo et al, "OE-A roadmap for organic and printed electronics". In Applications of Organic and Printed Electronics (pp. 1-26). Springer (2013)

[3] J. M. Deen, "Flexible electronics - opportunities and challenges". Proc EDSSC, pp. 1-2, (2013)

[4] S. Jacob et al, "Large area sensing surfaces: Flexible organic printed interfacing circuits and sensors", Proc. IEDM (pp. 19-24). (2015)

[5] H. Kang et al, "Bulk-Heterojunction Organic Solar Cells: Five Core Technologies for Their Commercialization". Adv. Mater. DOI: 10.1002 /adma.201601197 (2016)

[6] J. Zhao et al, "Efficient organic solar cells processed from hydrocarbon solvents" Nat. Ener. 1, 15027 (2016)

[7] P. B. Lechêne et al, "Organic solar cells and fully printed supercapacitors optimized for indoor light energy harvesting". Nano Energy, 26, p.631, (2016).

[8] J. M. Verilhac, "Recent developments of solution-processed organic photodetectors". Eur. Phys. J. Appl. Phys., 63(1), 14405 (2012)

[9] S. F. Tedde et al "Imaging with organic and hybrid photodetectors". Proc. IEDM 2014.

[10] G. Pace et al, "Printed photodetectors". Semi. Sci. Tech, 30, (2015).

[11] R. A. Janssen et al, "Factors limiting device efficiency in organic photovoltaics". Adv. Mater., 25(13), 1847-1858 (2013).

[12] C. Yi et al. "Perovskite Photovoltaics With Outstanding Performance Produced by Chemical Conversion of Bilayer Mesostructured Lead Halide/TiO2 Films” Adv. Mater. 28(15), 2964-2970, (2016).

[13] M. Mori et al, "Thin organic photoconductive film image sensors with extremely high saturation of 8500 electrons $/ \mu \mathrm{m}^{2}$," in Symp. VLSI technology, pp. 22-23 (2013).

[14] A. Pierre et al. "High Detectivity All-Printed Organic Photodiodes". Adv. Mater., 27(41), 6411-6417 (2015).

[15] E. Saracco et al. "Work function tuning for high-performance solution- 
processed organic photodetectors with inverted structure" Adv. Mater., vol. 25(45), 6534-6538, (2013).

[16] J. C. Blakesley et al, "Modeling the imaging performance of prototype organic x-ray imagers" Med. Phys. 35, p. 225 (2008)

[17] C. H. Peters et al, "High efficiency polymer solar cells with long operating lifetimes" Adv. Ener. Mater., 1(4), 491-494 (2011).

[18] P. Cheng et al, "Stability of organic solar cells: challenges and strategies”. Chem. Soc. Rev., 45(9), 2544-2582 (2016).

[19] H. Bässler et al, "Charge transport in organic semiconductors". (pp. 165). Springer Berlin Heidelberg (2011).

[20] Y. Tsutsui et al, "Unraveling Unprecedented Charge Carrier Mobility through Structure Property Relationship of Four Isomers of C12-BTBTC12". Adv. Mat., DOI: 10.1002/adma.201601285 (2016).

[21] S. Fratini, "The transient localization scenario for charge transport in crystalline organic materials". Adv. Funct. Mater. 26(14), (2016)

[22] T. Sakanoue et al, "Band-like temperature dependence of mobility in a solution-processed organic semiconductor". Nat. Mater, 9(9), 736-740 (2010).

[23] H. Bässler, "Charge transport in disordered organic photoconductors a Monte Carlo simulation study". Phys. Stat. Sol. (b), 175(1), 15-56 (1993).

[24] C. Jungemann et al, "DC, AC and noise simulation of organic semiconductor devices based on the master equation", Proc. SISPAD 2014

[25] P. Kordt et al. "Modeling of organic light emitting diodes: From molecular to device properties". Adv. Func. Mater., 25(13), 1955-1971 (2015).

[26] W. F. Pasveer et al, "Unified description of charge-carrier mobilities in disordered semiconducting polymers",Phys. Rev. Lett. 94(20), 206601 (2005).

[27] A. J. Mozer et al, "Negative electric field dependence of charge carrier drift mobility in conjugated semiconducting polymers". Chem. Phys. Lett., 389(4), 438-442 (2004).

[28] L. Meng et al, "An improved dynamic Monte Carlo model coupled with Poisson equation to simulate the performance of organic photovoltaic devices". J. Chem. Phys., 134(12), 124102 (2011).

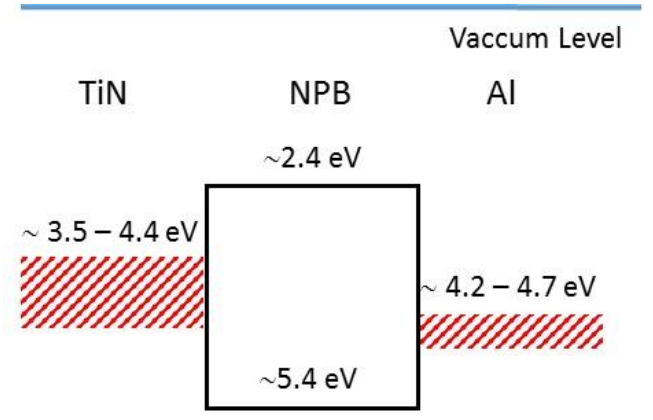

Fig. 1 : Band Diagram of the p-only asymmetrical $\alpha$-NPB device

[29] C. Groves et al., "Bimolecular recombination in polymer electronic devices" Phys. Rev. B, 78(15), 155205 (2008).

[30] W. Brütting et al, "Device physics of organic light-emitting diodes based on molecular materials". Org. Electron. 2(1), 1-36 (2001)

[31] M. Kröger et al, "P-type doping of organic wide band gap materials by transition metal oxides" Org. Electron, 10(5), 932-938 (2009).

[32] M. Hack, et al, "Theoretical modeling of amorphous silicon-based alloy p-i-n solar cells”. J. Appl. Phys. 54(10), 5858-5863 (1983).

[33] M. S. Lundstrom et al, "Numerical analysis of heterostructure semiconductor devices". IEEE Trans. Electron Device 30(9), 1151-1159 (1983).

[34] D. Sievers et al, "Modeling optical effects and thickness dependent current in polymer BHJ solar cells". J. Appl. Phys. 100(11), 114509 (2006)

[35] P. Stallinga, "Electronic transport in organic materials: comparison of band theory with percolation/hopping theory". Adv. Mater, 23(30), p. 3356 (2011).

[36] B. Popescu et al, "Modeling and simulation of organic photodetectors for low light intensity applications". IEEE Trans. Electron Device, 60, p.1975 (2013).

[37] B. Ray "Random vs regularized OPV: Limits of performance gain of organic bulk heterojunction solar cells by morphology engineering" Sol. Ener. Mat. Sol. Cells, 99, 204-212 (2012).

[38] B. Bouthinon et al. "Impact of blend morphology on interface state recombination in bulk heterojunction organic solar cells". Adv. Func. Mater., 25(7), 1090-1101 (2015)

[39] W. Tress, "Influence of Hole-Transport Layers and Donor Materials on Open-Circuit Voltage and Shape of I-V Curves of Organic Solar Cells". Adv. Funct. Mater. 21(11), 2140-2149 (2011)

[40] J. Bisquert et al "Theory of impedance and capacitance spectroscopy of solar cells with dielectric relaxation, drift-diffusion transport, and recombination". J. Phys. Chem. C, 118(33), 18983-18991 (2014)

[41] A. Seemann et al "Reversible and irreversible degradation of organic solar cell performance by oxygen". Sol. Energy, 85(6), 1238-1249 (2011).

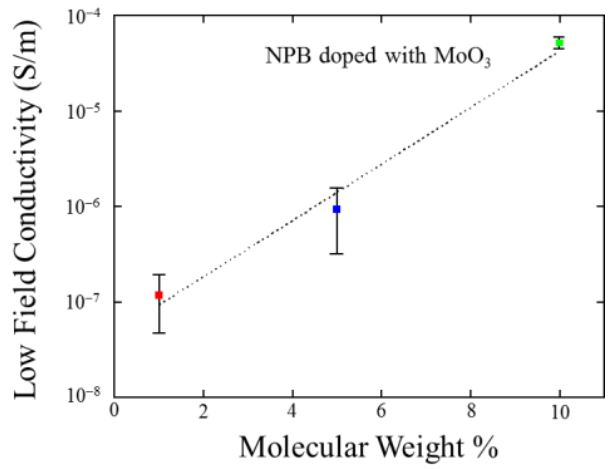

Fig. 2 : Low-field conductivity extracted from I-V experiments on each device versus nominal molecular weight (error bar indicates variability)

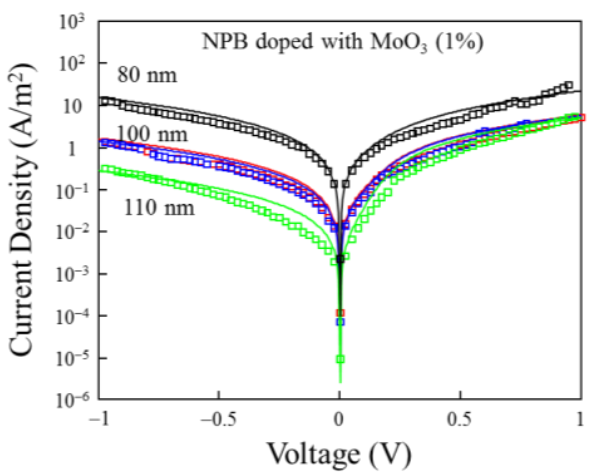

Fig. 3 : I-V curves of doped $\alpha$-NPB (1\%) symbols $=$ experiments, line $=$ simulations.

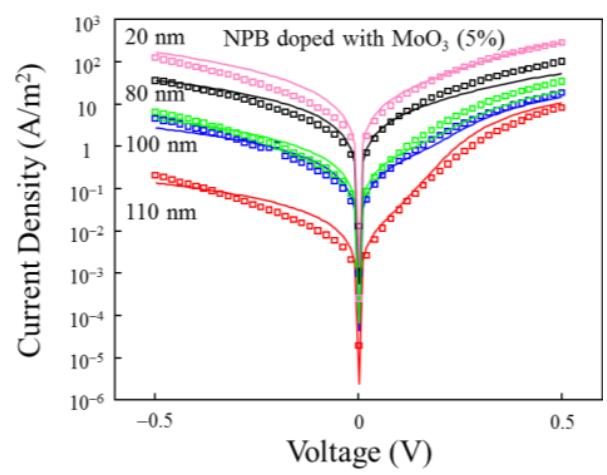

Fig. 4 : I-V curves of doped $\alpha-N P B(5 \%)$ symbols $=$ experiments, line $=$ simulations.

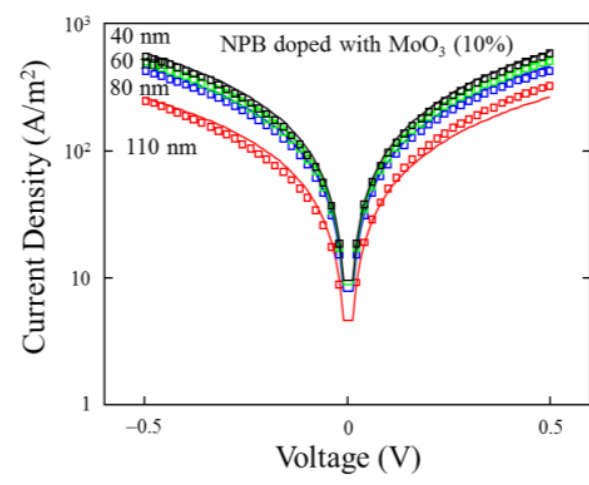

Fig. 5 : I-V curves of doped $\alpha$-NPB (10\%) symbols $=$ experiments, line $=$ simulations. 


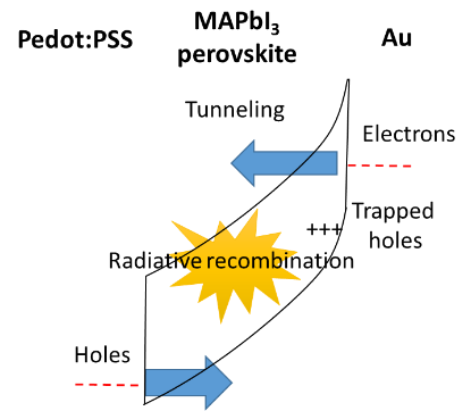

Fig. 6 : Schematic band diagram of the p-only polycrystalline perovskite $\mathrm{MAPbI}_{3}$

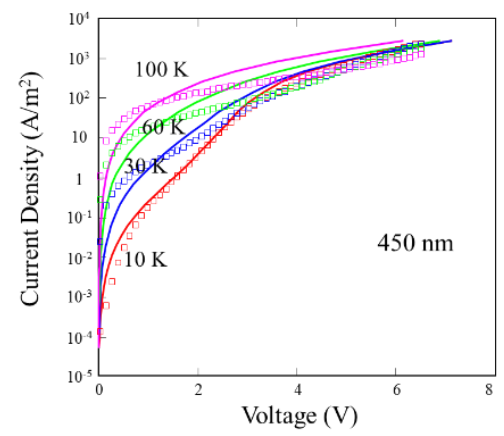

Fig. 8 : I-V curves for several temperature of the device of Fig. 6. Simulations are in good agreement with experiments, but do not perfectly capture the thermally activated low field regime.

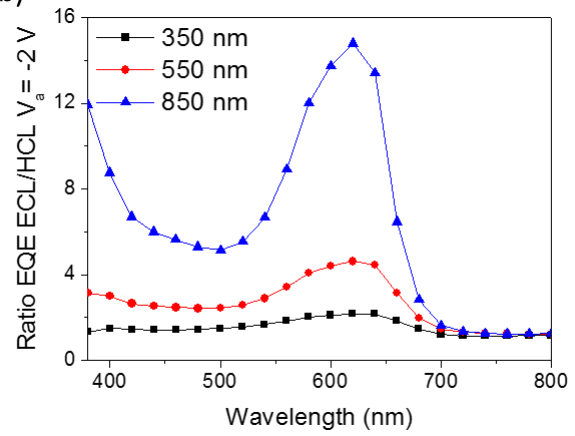

Fig. 10 : Experimental ratio between the back side external quantum efficiency $\mathrm{EQE}$ and the front side @ $-2 \mathrm{~V}$ versus wavelength

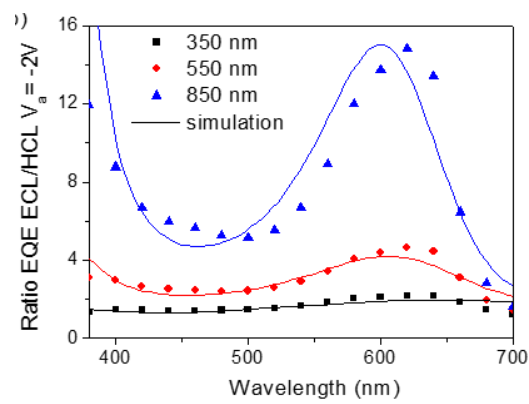

Fig. 12 : Same as Fig. 10, including simulations where an acceptor deep traps has been introduced (same concentration for all curves)

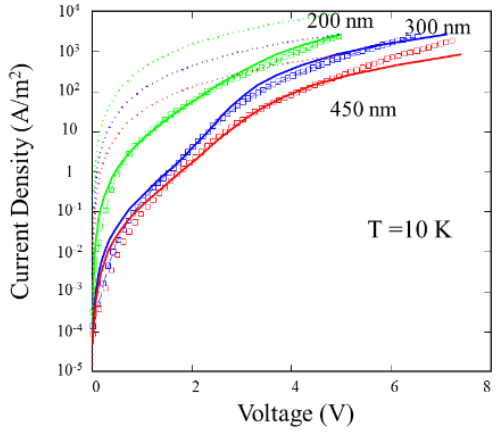

Fig. 7 : I-V curves @ $10 \mathrm{~K}$ of the device of Fig. 6. symbols = experiments, line $=$ simulations, dotted line model without trapping.

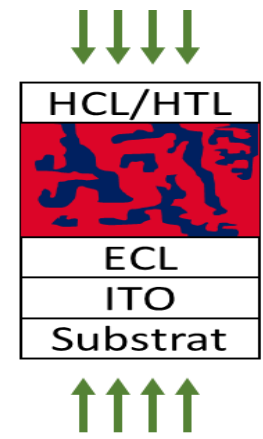

Fig. 9 : Composition of the semitransparent organic photodiode, lighted by either the back side (substrate) or the front side.

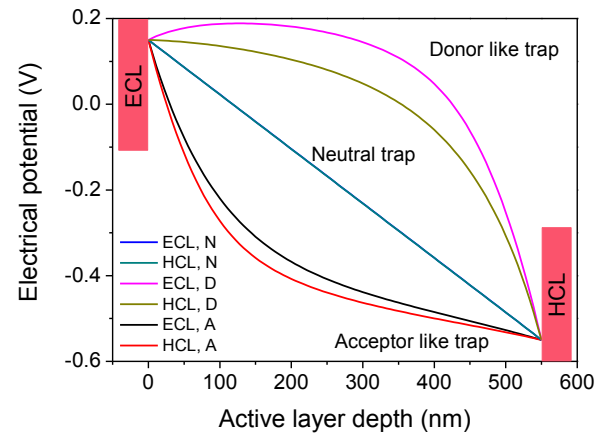

Fig. 11 : Simulation of the impact of negatively charged, positively and neutral volume traps (acceptors) on the electrical potential

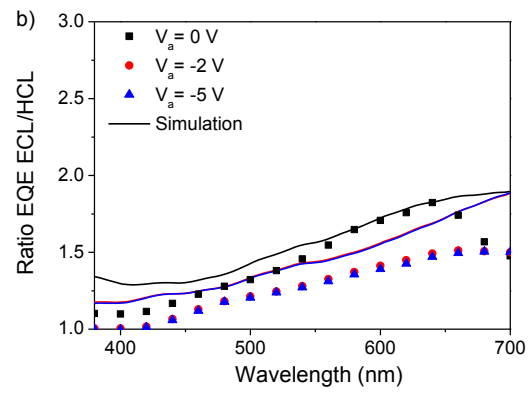

Fig. 13 : Same as Fig. 12, in absence of traps in simulations, where oxygen has been removed from experiments 